www.nature.com/pj

\title{
Estimation of the hydrodynamic screening length in $\kappa$-carrageenan solutions using NMR diffusion measurements
}

\author{
Qiuhua Zhao and Shingo Matsukawa
}

We quantify the hydrodynamic screening length $(\xi)$ in $\kappa$-carrageenan solutions by observing restrictions on the diffusion of several pullulan probes, each with a different molecular weight $\left(M_{\mathrm{w}}\right)$. The diffusion coefficient of pullulan in a pure solvent $\left(D_{\text {pull, }, 0}\right)$ and a $\kappa$-carrageenan solution $\left(D_{\text {pull }}\right)$ were measured using a pulsed field gradient stimulated spin-echo ${ }^{1} \mathrm{H}$ nuclear magnetic resonance. The hydrodynamic radii $\left(R_{\mathrm{H}}\right)$ of the pullulan samples were determined using $D_{\text {pull,o }}$ measured at $25^{\circ} \mathrm{C}$ and the Stoke-Einstein equation. The echo signal intensity of $\kappa$-carrageenan without a gradient, $I_{\text {kappa }}(0)$, decreased steeply at around the sol-to-gel transition temperature $\left(T_{\mathrm{sg}}\right)$, suggesting that the $\kappa$-carrageenan chains formed helical aggregates at $T_{\mathrm{sg}}$ diluting the solute $\kappa$-carrageenan concentration. The ratio, $D_{\text {pull }} / D_{\text {pull, }, 0}$, was less than 1 , indicating that the mobility of pullulan was restricted by the $\kappa$-carrageenan chains. Over the entire temperature range, pullulan with larger molecular size yielded more restrictions on the molecular mobility, and $D_{\text {pull }} / D_{\text {pull, }, 0}$ decreased as the $M_{\mathrm{w}}$ of pullulan increased. Below $T_{\mathrm{sg}}, D_{\text {pull }} / D_{\text {pull,0}}$ increased as the temperature decreased. This behavior suggests that a decrease in the restriction degree by the $\kappa$-carrageenan chains is concomitant with the formation of aggregates. To quantify the restriction degree, $\xi$ was estimated at various temperatures using the relation $D_{\text {pull }} / D_{\text {pull, } 0}=\exp \left(-R_{\mathrm{H}} / \xi\right)$.

Polymer Journal (2012) 44, 901-906 doi:10.1038/pj.2012.107; published online 13 June 2012

Keywords: diffusion coefficient; gel; gradient NMR; hydrodynamic radius; hydrodynamic screening length; pullulan; $\kappa$-carrageenan

\section{INTRODUCTION}

The diffusion of solutes in a gel matrix has an important role in applications such as the delivery of drugs and the encapsulation of fragrances. ${ }^{1,2}$ NMR has proved to be a powerful technique for determining the dynamic and structural properties of food model systems $^{3}$ and the gelation process of polysaccharides. ${ }^{4,5}$ NMR relaxation times give information about the tumbling motion of molecules in food hydrocolloid systems. The diffusion coefficients determined by pulsed-field-gradient NMR reflect the translational motion of molecules. The diffusion coefficient of solutes in a polymer matrix strongly depends on molecular weight, temperature, viscosity and the network structure of the diffusing matrix. Any factor that reduces the space delineated by the polymer chains will have an effect on the molecular mobility of the solute. In polysaccharide gels, the aggregation of polysaccharide chains can enlarge the diffusing space. Therefore, to understand the diffusion of molecules in a gel, it is important to understand the aggregation behavior.

$\kappa$-Carrageenan is a linear sulfated polysaccharide that is extracted from marine red algae. As shown in Figure 1, $\kappa$-carrageenan comprises two galactose units: $\alpha-(1-3)$-D-galactose-4-sulfate and $\beta-(1-4)-3,6$-anhydro-D-galactose. It is a gelling agent that has applications in the food industry, ${ }^{6}$ as well as the pharmaceutical ${ }^{7}$ and biotechnology sectors. ${ }^{8} \kappa$-Carrageenan in a hot aqueous solution in the presence of cations has the ability to form thermoreversible gels upon cooling. The gelation process of $\kappa$-carrageenan has been widely studied. ${ }^{9-11}$ The process upon cooling is commonly modeled as the transition of $\kappa$-carrageenan chains from a random coil to a helix conformation. This transition is followed by the aggregation of helices to form a network.

In the present study, our objectives were to investigate the diffusion behavior of several pullulan samples, each with a different molecular weight, in the $\kappa$-carrageenan solutions and to use the observed restrictions on the diffusion mobility of the pullulan probes to estimate the hydrodynamic mesh size of the $\kappa$-carrageenan solutions.

\section{EXPERIMENTAL PROCEDURE}

Materials and sample preparation

The $\kappa$-carrageenan was purchased from Sigma Chemical Co. (St Louis, MO, USA) and was used without any purification. The concentrations of $\mathrm{K}^{+}, \mathrm{Na}^{+}$ and $\mathrm{Ca}^{2+}$ in the original samples were $6.8 \%, 0.6 \%$ and $2.4 \%$, respectively. No excess salt was added during sample preparation. The molecular weights of pullulan sample purchased from Showa Denko Co. (Tokyo, Japan) were 


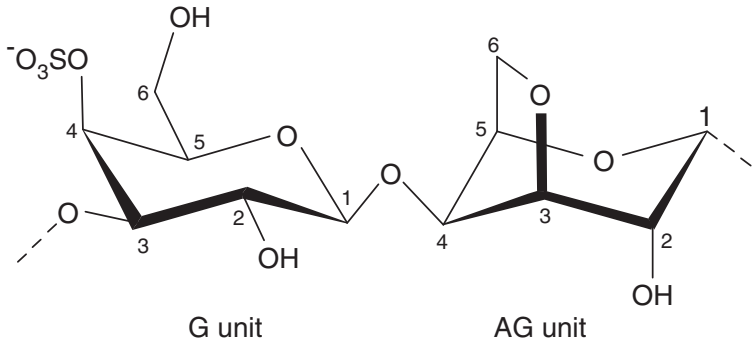

Figure 1 Idealized disaccharide repeating unit of $\kappa$-carrageenan.

$2.28 \times 10^{4}, 4.73 \times 10^{4}, 10.7 \times 10^{4}, 21.2 \times 10^{4}$ and $40.4 \times 10^{4} \mathrm{~g} \mathrm{~mol}^{-1}$. Each sample contained one of the pullulan probes and was prepared as follows: powdered $\kappa$-carrageenan was dispersed in $\mathrm{D}_{2} \mathrm{O}$ by stirring for $1 \mathrm{~h}$ at room temperature and $0.5 \mathrm{~h}$ at $80^{\circ} \mathrm{C}$. Thereafter, an equivalent amount of $0.2 \%$ pullulan $/ \mathrm{D}_{2} \mathrm{O}$ solution was added. The mixture was stirred at $80^{\circ} \mathrm{C}$ for $0.5 \mathrm{~h}$ to obtain a homogeneous solution. Five solutions, each with a different molecular weight pullulan, were prepared using the same procedure and contained $1 \% \mathrm{~K}$ carrageenan and $0.1 \%$ pullulan. The concentrations of $\kappa$-carrageenan and pullulan are expressed in $\mathrm{w} / \mathrm{w} \%$ of $\kappa$-carrageenan and pullulan relative to the total weight of the solution. The resulting solutions were immediately transferred into a preheated $10 \mathrm{~mm}$ NMR tube. Samples used to measure the diffusion coefficient in a pure solvent were prepared using powdered pullulan mixed with $\mathrm{D}_{2} \mathrm{O}$ and stirred at $80^{\circ} \mathrm{C}$ for $0.5 \mathrm{~h}$

\section{NMR measurements}

Self-diffusion coefficient measurements using the pulsed field gradient stimulated spin-echo pulse sequence were performed on a Bruker Avance II 400WB spectrometer (Bruker Biospin GmbH, Rheinstetten, Germany) that was equipped with a gradient probe. A schematic diagram of the pulse sequence can be found in the literature. ${ }^{12}$ The temperature was controlled using a Bruker BVT-3200 (Bruker Biospin GmbH, Wissembourg, France) and was monitored using an optical fiber thermometer (Takaoka Electric Manufacturing Co., Tokyo, Japan) placed in the sample tube. Each measurement was carried out after waiting for $30 \mathrm{~min}$ to reach the thermal equilibrium.

The diffusion coefficients were determined using the following relationship between the echo signal intensities and the field-gradient parameters:

$$
I\left(2 \tau_{2}+\tau_{1}, g \delta\right)=I\left(2 \tau_{2}+\tau_{1}, 0\right) \exp \left[-\gamma^{2} \delta^{2} g^{2}(\Delta-\delta / 3) D\right]
$$

where $I\left(2 \tau_{2}+\tau_{1}, g \delta\right)$ and $I\left(2 \tau_{2}+\tau_{1}, 0\right)$ are the echo signal intensities at $t=2$ $\tau_{2}+\tau_{1}$ with and without the field gradient pulse, respectively; $\gamma$ is the gyromagnetic ratio of ${ }^{1} \mathrm{H} ; \delta$ is the duration of the gradient pulses; $g$ is the field gradient strength; and $\Delta$ is the diffusion time that corresponds to the time interval between two field gradient pulses. Note that $I\left(2 \tau_{2}+\tau_{1}, 0\right)$ has decayed from the initial intensity, $I(0,0)$, (that is, the signal intensity immediately after the first r. f. $2 / \pi$ pulse) by the relaxations of $T_{1}$ and $T_{2}$ :

$$
I\left(2 \tau_{2}+\tau_{1}, 0\right)=I(0,0) / 2 \exp \left[-2 \tau_{2} / T_{2}-\tau_{1} / T_{1}\right]
$$

where a decrease in the relaxation times causes a decrease in $I\left(2 \tau_{2}+\tau_{1}, 0\right)$.

In all experiments, $\delta$ and $\Delta$ were fixed to 1 and $10 \mathrm{~ms}$, respectively. The field gradient strength, $g$, was varied in eight steps within the range of $1.8-12.0 \mathrm{Tm}^{-1}$, the maximum value varied depending on the diffusing system. The $\pi / 2$ pulse length for ${ }^{1} \mathrm{H}$ was $16 \mu$ s and the relaxation delay was set to $3.5 \mathrm{~s}$. All chemical shifts were referenced to the peak at 5.36 p.p.m., which is attributed to the anomeric proton of pullulan.

\section{RESULTS AND DISCUSSION}

Pulsed-field-gradient ${ }^{1} \mathrm{H}$ spectra of pullulan in a pure solvent and a $\kappa$-carrageenan solution

Figure $2 \mathrm{a}$ shows the stacked ${ }^{1} \mathrm{H}$ spectra of the $0.1 \%$ pullulan solution. The signal intensity of pullulan decayed with increasing $g$, reflecting the diffusional motion of pullulan in a pure solvent.
Figure $2 \mathrm{~b}$ shows the pulsed-field-gradient ${ }^{1} \mathrm{H}$ spectra of the $1 \%$ $\kappa$-carrageenan solution containing $0.1 \%$ pullulan at various temperatures. The peaks that were selected for the following analysis of pullulan and $\kappa$-carrageenan are indicated by arrows. Detailed information on the signal assignments of $\kappa$-carrageenan and pullulan can be found in the literature. ${ }^{13,14}$ The intensity of the peak from the anomeric proton of pullulan at 5.36 p.p.m., which is separated from the peaks of $\kappa$-carrageenan, decayed with increasing $g$ and indicates the diffusion of pullulan among the network structure formed by the $\kappa$-carrageenan chains.

On the basis of Equation (1), a semi-logarithmic plot of $I_{\text {pull }}(g)$ as a function of $\gamma^{2} \delta^{2} g^{2}(\Delta-\delta / 3)$ gives a straight line with a slope of $-D$, if the diffusant has a single diffusion process. The semi-logarithmic plots of $I_{\text {pull }}(g)$ at $25^{\circ} \mathrm{C}$ in the pure solvent and the $\kappa$-carrageenan solution, obtained from the results of Figure 2, are shown in Figure 3. The first spectra at the lowest gradient strength in the pure solvent contains the residual water peak and was not used for the diffusion analysis. The plots lie on a straight line, indicating that pullulan has a single-mode diffusion process in both the pure solvent and the $\kappa$-carrageenan solution. The diffusion coefficient of pullulan, $D_{\text {pull, }}$, is obtained by fitting Equation (1) to the experiment data. The diffusion coefficient of pullulan in the $\kappa$-carrageenan solution is smaller than that in pure $\mathrm{D}_{2} \mathrm{O}$. This result suggests that pullulan suffers restrictions from the $\kappa$-carrageenan chains, and thus has slower diffusional motion. The restriction effect ${ }^{15,16}$ on the molecular mobility of pullulan will be discussed in detail later.

\section{Diffusion mobility of pullulan in a pure solvent}

The convection effect was studied by exploring the dependence of $D_{\text {pull, } 0}$ on the diffusion time ( $\Delta$ ) for different molecular weights at 25 , 30 , and $40{ }^{\circ} \mathrm{C}$ by varying $\Delta$ from 10 to $40 \mathrm{~ms} .{ }^{16,17}$ Figure 4 shows an example of the dependence of the diffusion coefficient on $\Delta$ for the $0.1 \%$ solution of pullulan with $M_{\mathrm{w}}=10.7 \times 10^{4}$. As $\Delta$ increased, the apparent $D$ did not increase at $25^{\circ} \mathrm{C}$; it increased slightly at $30{ }^{\circ} \mathrm{C}$ and visibly at $40^{\circ} \mathrm{C}$. These results indicate that the convection effect is significant at higher temperatures. For each temperature, $D_{\text {pull, } 0}$ can be determined by extrapolating the plot to $\Delta=0 \mathrm{~ms}$. Note that the experimental data was more scattered at $40^{\circ} \mathrm{C}$ than at the other two temperatures. As a result, the extrapolation is inaccurate at $40{ }^{\circ} \mathrm{C}$ and the inaccuracy is expected to increase with increasing temperature. Therefore, the temperature dependence of $D_{\text {pull, } 0}$ was calculated using the $D_{\text {pull, } 0}$ measured at $25^{\circ} \mathrm{C}$ and Stoke-Einstein equation ${ }^{18}$ as described below.

Figure 5 shows the molecular weight dependence of $D_{\text {pull, } 0, T^{\circ}}$ $\left(D_{\text {pull,0 }}\right.$ at $\left.25^{\circ} \mathrm{C}\right)$, where $T^{0}$ represents the temperature in Kelvin, $298 \mathrm{~K}$. The relationship between the molecular weight, $M_{\mathrm{w}}$, and the diffusion coefficient in a dilute solution, $D_{0}$, can be expressed as: ${ }^{19}$

$$
D_{0}=a M_{\mathrm{w}}^{-v}
$$

where $a$ is a constant related to the segment size of the polymer chain and $v$ is an exponent that depends on the polymer-solvent system. As seen in Figure 5, the double-logarithmic plot of $D_{\text {pull, } 0, \mathrm{~T}^{\circ}}$ as a function of $M_{\mathrm{w}}$ is essentially linear, and the experimental result gives the following equation:

$$
D_{\text {pull }, 0, \mathrm{~T}^{\mathrm{o}}}=2.25 \times 10^{-8} M_{\mathrm{w}}^{-0.59}\left(\mathrm{~m}^{2} \mathrm{~s}^{-1}\right) .
$$

The value of the exponent, $v$, was found to be 0.59 that is close to that for flexible polymers in a good solvent. The experimental results obtained by Nisihinari et al. ${ }^{20}$ yielded a $v$ equal to 0.51 . The diffrence in $v$ is most likely attributed to the method used to determine $D_{\text {pull }, 0, \mathrm{~T}^{\circ}}$. Nisihinari et al. used the intercept of the concentration 


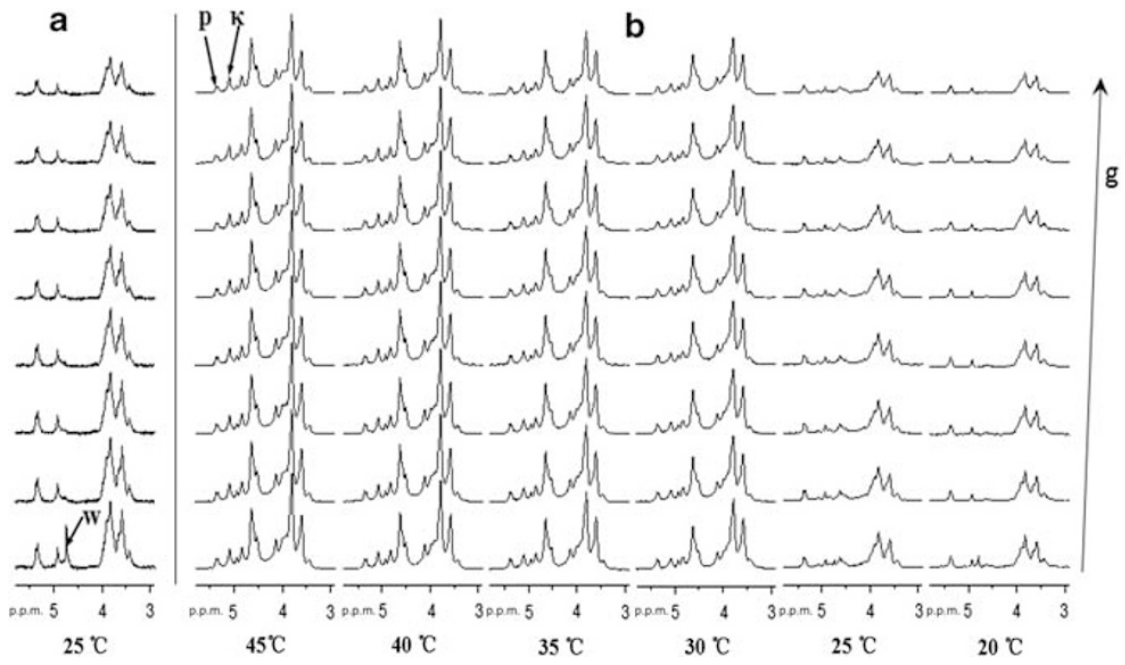

Figure 2 (a) Stacked pulsed field gradient stimulated spin-echo (PGSTE) ${ }^{1} \mathrm{H}$ spectra of a $0.1 \%$ solution of pullulan with $M_{\mathrm{w}}=10.7 \times 10^{4} \mathrm{~g} \mathrm{~mol}{ }^{-1}$ at $25^{\circ} \mathrm{C}$ as a function of the field gradient strength $g$ ( $g$ was varied from 1.8 to $6.0 \mathrm{~T} \mathrm{~m}^{-1}$ in the vertical direction). The residual water peak is indicated by a ' $w$ '. (b) Stacked PGSTE ${ }^{1} \mathrm{H}$ spectra of a $1 \% \kappa$-carrageenan solution containing $0.1 \%$ pullulan with $M_{\mathrm{w}}=10.7 \times 10^{4} \mathrm{~g} \mathrm{~mol}{ }^{-1}$ as a probe polymer during the cooling process. The peaks selected for the diffusion analysis of the pullulan and $\kappa$-carrageenan are indicated by ' $\mathrm{p}$ ' and ' $\kappa$ ', respectively. The field gradient strength, $g$, was varied from 2.0 to $7.0 \mathrm{~T} \mathrm{~m}^{-1}$ in the vertical direction.

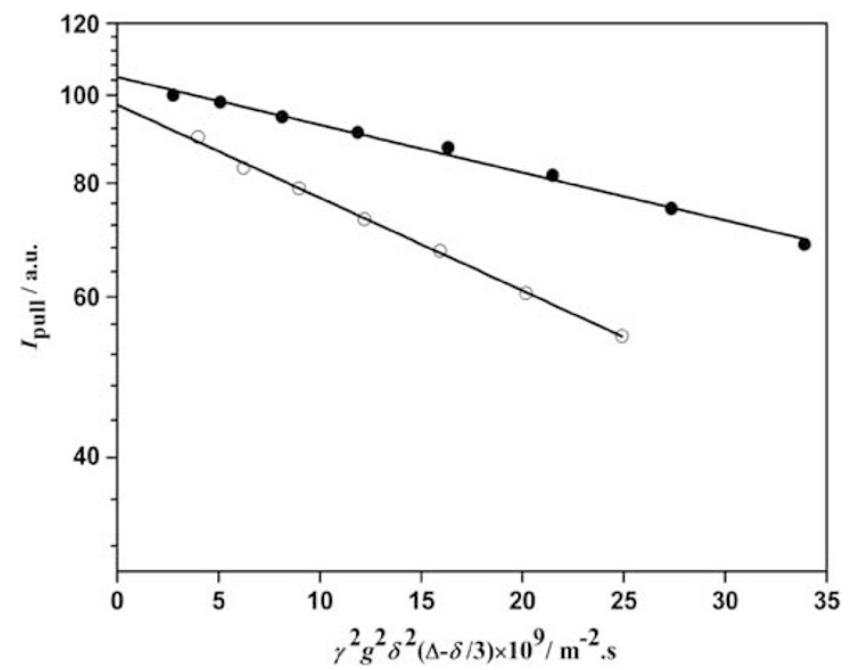

Figure 3 The signal decay of pullulan $\left(M_{w}=10.7 \times 10^{4} \mathrm{~g} \mathrm{~mol}^{-1}\right)$ in a $1 \%$ $\kappa$-carrageenan solution $(\bullet)$ and a pure solvent $(O)$ at $25^{\circ} \mathrm{C}$. Because the band selected for the analysis (full spectra) overlapped the residual water peak (Figure 2a), the first spectra at the lowest gradient $\left(1.8 \mathrm{~T} \mathrm{~m}^{-1}\right)$ was excluded from the calculation of the diffusion coefficient of the pullulan in a pure solvent. The lines are fits of Equation (1) to the data.

dependence of the pullulan diffusion coefficient at $c \rightarrow 0$ using photon correlation spectrometry. However, the exact reasons for the deviation of $v$ are not yet clear.

Even for the pullulan sample with highest molecular weight, the concentration of pullulan used in the present study $(0.1 \%)$ is well below the overlap concentration $\boldsymbol{c}^{\star}\left(\boldsymbol{c}^{\star} \approx 0.8 \%, \boldsymbol{c}^{\star}=\frac{M_{\mathrm{w}} / \mathrm{N}_{\mathrm{A}}}{(4 \pi / 3) R_{\mathrm{H}}^{3}}\right.$, and $N_{\mathrm{A}}$ is the Avogadro constant). ${ }^{21}$ It is also suggested that pullulan molecules behave as flexible random coils in the molecular weight range of 20000 to $80000 .^{22}$ In this study, the molecular weight varied from 22800 to 404000 . Under these conditions, the diffusivity of

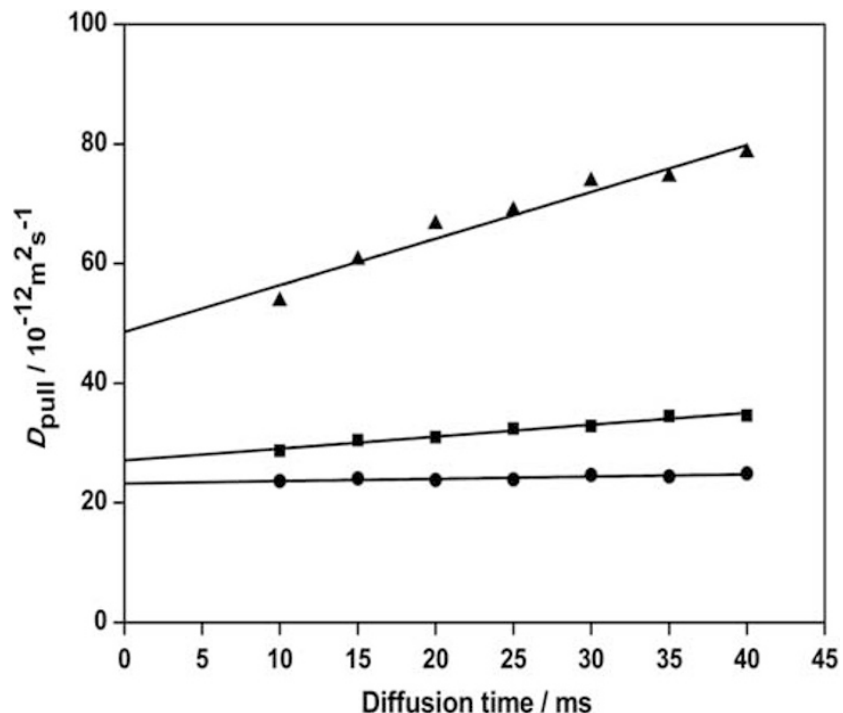

Figure 4 The diffusion time dependence of the diffusion coefficients of pullulan with $M_{\mathrm{w}}=10.7 \times 10^{4} \mathrm{~g} \mathrm{~mol}^{-1}$ in a $0.1 \%$ pullulan solution at $25^{\circ} \mathrm{C}$ (-), $30^{\circ} \mathrm{C}(\boldsymbol{\square})$ and $40^{\circ} \mathrm{C}(\mathbf{\Lambda})$.

pullulan can be described using Stokes-Einstein equation. The hydrodynamic radius, $R_{\mathrm{H}, \mathrm{T}}$, can be determined from $D_{\text {pull, }, \mathrm{T}}$ using:

$$
R_{\mathrm{H}, \mathrm{T}}=\frac{k_{\mathrm{B}} T}{6 \pi \eta_{\mathrm{T}} D_{\text {pull }, 0, \mathrm{~T}}}
$$

where $k_{\mathrm{B}}$ is Boltzmann's constant, $T$ is the absolute temperature, $\eta_{\mathrm{T}}$ is the viscosity of heavy water and $\mathrm{T}$ in the subscript of $D$ means the value of the diffusion coefficient at the temperature $T$. We assumed that $R_{\mathrm{H}, \mathrm{T}}$ is a constant in the temperature range of the measurements. The constant value, $\overline{R_{\mathrm{H}}}$, should be identical 


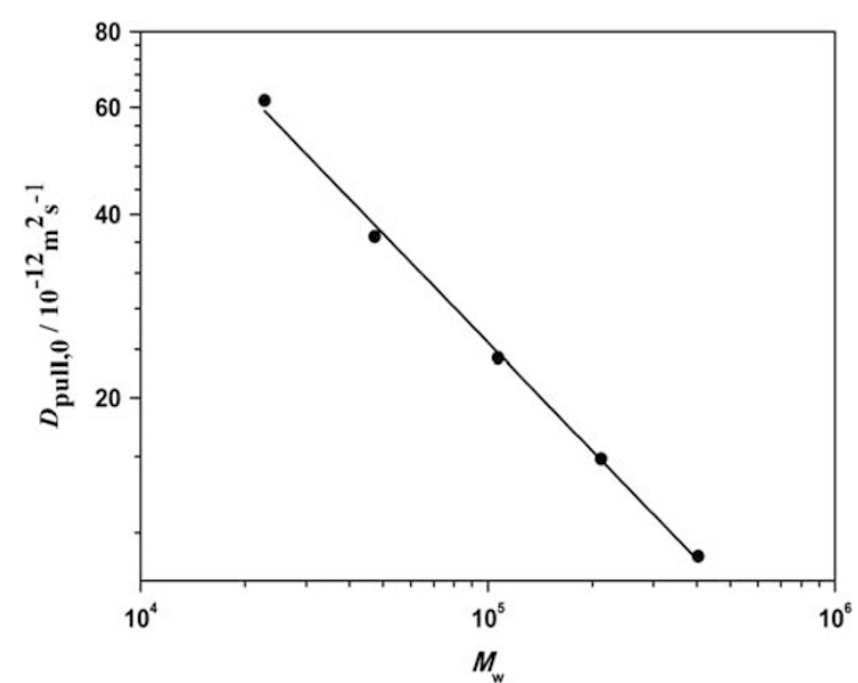

Figure 5 Double-logarithmic plot of the diffusion coefficient of pullulan in $\mathrm{D}_{2} \mathrm{O}, D_{\text {pull, }}$, as a function of the molecular weight, $M_{\mathrm{w}}$, of pullulan at $25^{\circ} \mathrm{C}$ (-). The line is a fit of Equation (3) to the data: $D_{0}=a M_{\mathrm{w}}{ }^{v}$.

to $R_{\mathrm{H}, \mathrm{T}^{\circ}}$ for each of the pullulan samples, which are calculated from their respective $D_{\text {pull, }, 0, T^{\circ}}$ using Equation (5). The value of $D_{\text {pull }, 0, \mathrm{~T}^{\circ}}$ can be determined from $M_{\mathrm{w}}$ using Equation (4). The following equation is obtained:

$$
\overline{R_{\mathrm{H}}}=R_{\mathrm{H}, \mathrm{T}^{\mathrm{o}}}=\frac{k_{\mathrm{B}} T^{\mathrm{o}}}{6 \pi \eta_{\mathrm{T}^{\mathrm{o}}} D_{\text {pull }, 0, \mathrm{~T}^{\mathrm{o}}}}=8.82 \times 10^{-3} M_{\mathrm{w}}^{0.59}(\mathrm{~nm}) .
$$

Thus, $\overline{R_{\mathrm{H}}}$ of pullulan is determined from the molecular weight.

Because of the effect of convection, it is difficult to obtain an accurate value for $D_{\text {pull, } 0}$ at high temperatures. Therefore, the values of $D_{\text {pull, }, \mathrm{T}}$ at various temperatures were calculated using $D_{\text {pull, }, 0, \mathrm{~T}^{\circ}}$ as follows:

$$
D_{\text {pull }, 0, \mathrm{~T}}=\frac{k_{\mathrm{B}} T}{6 \pi \eta_{\mathrm{T}} \overline{R_{\mathrm{H}}}}=D_{\text {pull }, 0, \mathrm{~T}^{\mathrm{o}}} \times \frac{T / \eta_{\mathrm{T}}}{T^{\mathrm{o}} / \eta_{\mathrm{T}^{\mathrm{o}}}} .
$$

To estimate the restrictions on the mobility of pullulan in the $\kappa$ carrageenan solution, the calculated $D_{\text {pull, }, 0, T}$ were compared with the diffusion coefficients of pullulan in the $\kappa$-carrageenan solution, $D_{\text {pull, }}$, as described in the following section.

\section{The structural changes in the $\kappa$-carrageenan solution during gelation}

The temperature dependence of the echo signal intensities of $\kappa$ carrageenan without a gradient $I_{\text {kappa }}(0)$ was determined from the intercept of the fit of Equation (1) to $I_{\text {kappa }}(g)$ as a function of $\gamma^{2} \delta^{2} g^{2}(\Delta-\delta / 3)$. The temperature dependence is shown in Figure 6, where the $I_{\text {kappa }}(0)$ were normalized based on $I_{\text {kappa }}(0)$ at $50^{\circ} \mathrm{C}$. The peak in the ${ }^{1} \mathrm{H}$ NMR spectrum at 5.17 p.p.m. (Figure $2 b$ ) for the proton at position 1 on the $\beta$-(1-4)-3,6-anhydro-D-galactose unit (Figure 1) of $\kappa$-carrageenan ${ }^{14}$ was used for the diffusion analysis. At high temperature, $I_{\text {kappa }}(0)$ decreased slightly with decreasing temperature, which is most likely a result of an increase in the viscosity upon cooling, the relaxation time, $T_{1}$ and $T_{2}$, values were thus decreased and led to a decrease in $I_{\text {kappa }}(0)$ according to Equation (2). At the sol-to-gel transition temperature $T_{\text {sg }}\left(28^{\circ} \mathrm{C}\right.$, determined by falling ball method), ${ }^{12,23} I_{\text {kappa }}(0)$ decreased sharply. This decrease in $I_{\text {kappa }}(0)$ corresponds to a decrease in the solute $\kappa$-carrageenan chains and an increase of the helical aggregates, which

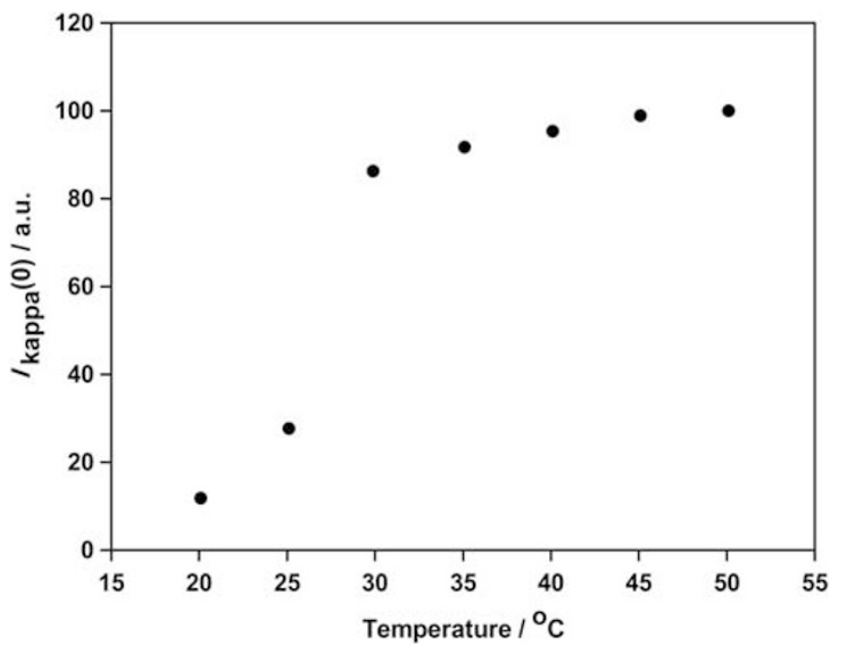

Figure 6 The temperature dependence of the echo signal intensity of $\kappa$-carrageenan without a gradient $I_{\text {kappa }}(0)$ during the cooling process. The $I_{\text {kappa }}(0)$ were determined using Equation (1).

has strongly restricted molecular motion and a short relaxation time. ${ }^{12,24}$ The changing tendency of the diffusion coefficient for $\kappa$-carrageenan, $D_{\text {kappa }}$, indicates that $D_{\text {kappa }}$ increased markedly as the temperature decreased below $T_{\mathrm{sg}}$ (data not shown). The inclusion of the $\kappa$-carrageenan chains into aggregates is thought to decrease the local friction through the hydrodynamic interaction and will lead to an increase in the molecular diffusion in the interspace. Nonetheless, $D_{\text {kappa }}$ increased more than expected from the decrease in the local friction. A GPC measurement shows that the $\kappa$-carrageenan used in this study has a wide molecular weight distribution, namely, the $\kappa$-carrageenan chains consist of long and short chains. From these results, it is believed that the relatively short $\kappa$-carrageenan chains were left as solute among the aggregated bundles, because the aggregation tends to gather longer chains in priority. A similar behavior was confirmed for gellan and agarose. ${ }^{12,24}$ Finally, $I_{\text {kappa }}(0)$ at $20{ }^{\circ} \mathrm{C}$ was only approximately $10 \%$ of that at $50{ }^{\circ} \mathrm{C}$, reflecting an ability of $\kappa$-carrageenan to gel.

Molecular mobility of pullulan in a $1 \% \kappa$-carrageenan solution The dilution of the $\kappa$-carrageenan concentration in solute due to the formation of aggregates was expected to affect the mobility of the molecules existing in the interspaces of the network formed by the $\kappa$-carrageenan chains. To further elucidate this effect, several pullulan samples, each with a different molecular weight, were added to the $\kappa$-carrageenan solution as probe molecules. Their diffusion coefficients were measured at various temperatures. The results are shown in Figure $7 \mathrm{a}$. At high temperatures, $D_{\text {pull }}$ decreased slightly because the molecular mobility decreased with decreasing temperature. Below $T_{\text {sg }}, D_{\text {pull }}$ increased with decreasing temperature, indicating an increase in the molecular mobility. As discussed above, the $\kappa$-carrageenan chains were involved in aggregation near $T_{\mathrm{sg}}$. The formation of thick aggregated bundles and network structures by solute $\kappa$-carrageenan chains is thought to result in an increase in the network mesh size. ${ }^{12,16,24}$ The diffusion of pullulan is thought to be restricted by hydrodynamic interactions with the solute $\kappa$-carrageenan chains as well as the network of aggregates. Therefore, the restriction became smaller as the solute $\kappa$-carrageenan concentration decreased 


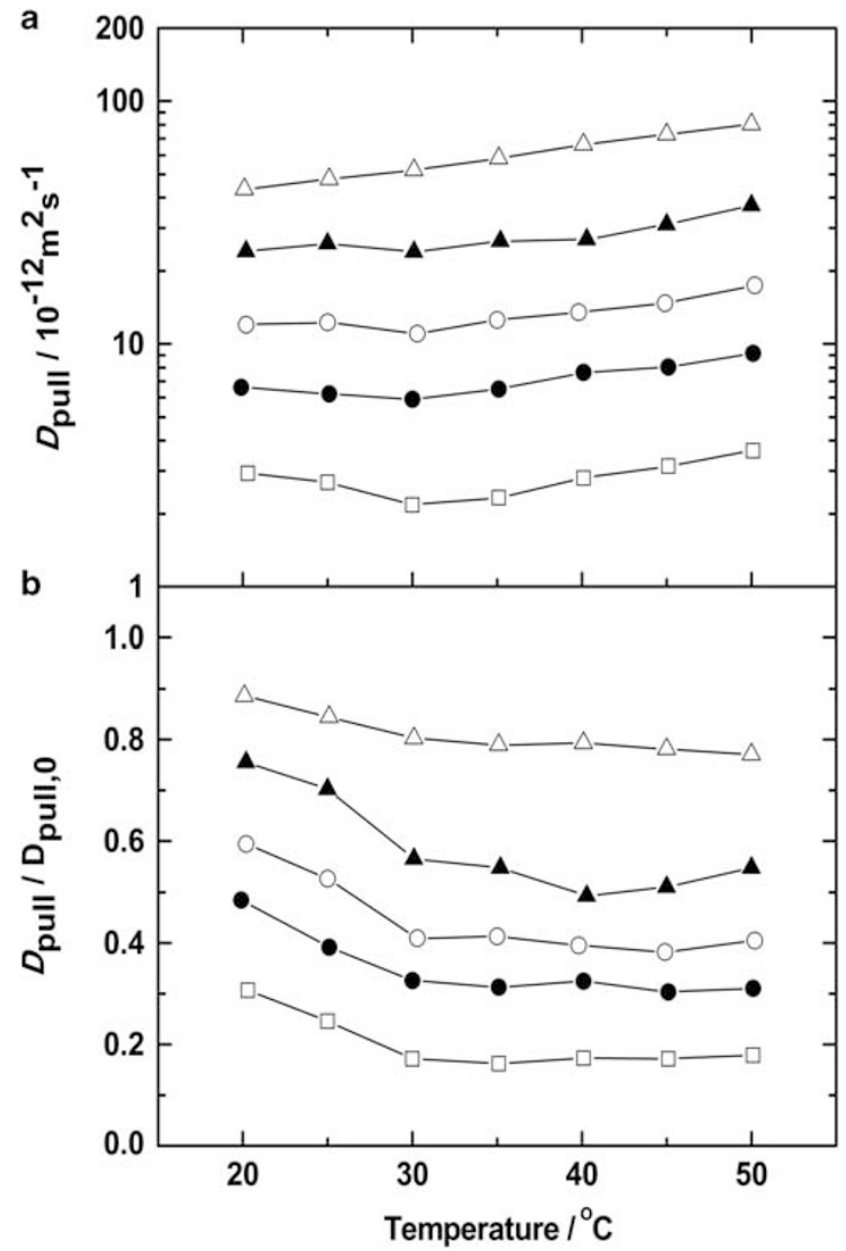

Figure 7 The temperature dependence of (a) the diffusion coefficients of pullulan, $D_{\text {pull; }}$ and (b) the ratio $D_{\text {pull }} / D_{\text {pull, }}$ in the $1 \% \kappa$-carrageenan solutions for pullulan with different molecular weights $\left(M_{\mathrm{w}}\right)$ : $40.4 \times 10^{4} \mathrm{~g} \mathrm{~mol}^{-1}(\square), 21.2 \times 10^{4} \mathrm{~g} \mathrm{~mol}^{-1}(\bullet), 10.7 \times 10^{4} \mathrm{~g} \mathrm{~mol}^{-1}(\bigcirc)$, $4.73 \times 10^{4} \mathrm{~g} \mathrm{~mol}^{-1}(\mathbf{\Delta})$ and $2.28 \times 10^{4} \mathrm{~g} \mathrm{~mol}^{-1}(\triangle)$. Lines are drawn only as a visual aid.

and the network mesh size increased, both of which result from the thickening of the aggregates upon further cooling.

\section{Calculation of hydrodynamic screening length in a $\kappa$-carrageenan} solution

As shown in Figure 7a, higher molecular weight pullulan is characterized by a stronger restriction from the $\kappa$-carrageenan chains. For a better understanding of how the $\kappa$-carrageenan chains restricted the diffusion of pullulan, the diffusion coefficients of pullulan $\left(D_{\text {pull }}\right)$ are divided by their values in a pure solvent $\left(D_{\text {pull, }, 0}\right)$. The ratios, $D_{\text {pull }} / D_{\text {pull, }, 0}$, are plotted as a function of temperature in Figure $7 \mathrm{~b}$. The ratio is less than 1 over the entire temperature range. This result clearly shows that the diffusion of pullulan in a $\kappa$-carrageenan solution and gel is restricted by hydrodynamic interactions. During the cooling process, $D_{\text {pull }} / D_{\text {pull, } 0}$ was nearly constant at high temperatures, and increased with decreasing temperature below $T_{\mathrm{sg}}$. This result suggests that the restriction on $D_{\text {pull }}$ by the $\kappa$-carrageenan chains decreased. Moreover, it is evident that a larger molecular weight of pullulan leads to a lower value of $D_{\text {pull }} / D_{\text {pull, }, 0}$, confirming that the restriction is higher for larger molecular weight pullulan. The restriction by the hydrodynamic interaction on the mobility can be

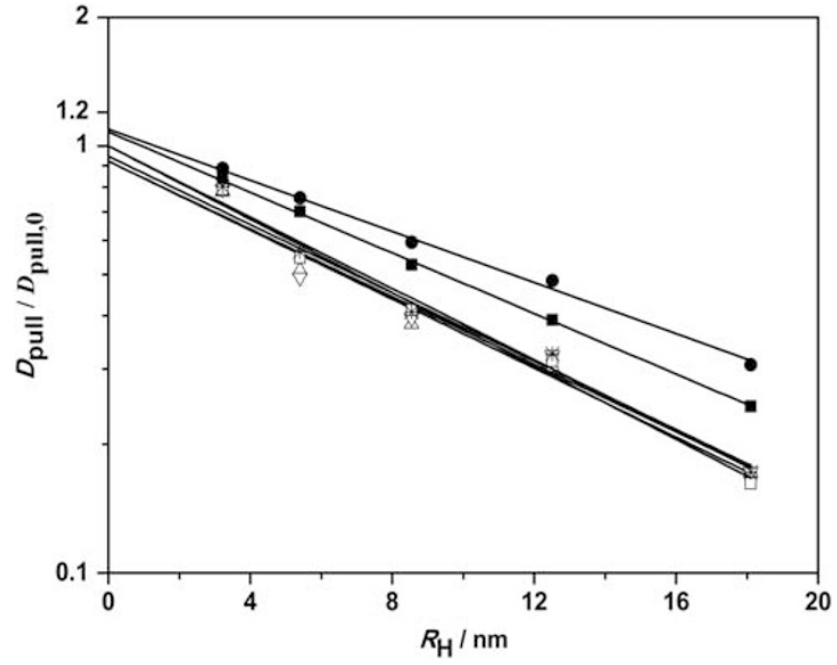

Figure 8 Semi-logarithmic plots of $D_{\text {pull }} / D_{\text {pull, }, 0}$ as a function of the hydrodynamic radius of pullulan, $R_{\mathrm{H}}$, at $20^{\circ} \mathrm{C}(\bullet), 25^{\circ} \mathrm{C}(\boldsymbol{\square}), 30^{\circ} \mathrm{C}(*)$, $35^{\circ} \mathrm{C}(\square), 40^{\circ} \mathrm{C}(\nabla), 45^{\circ} \mathrm{C}(\triangle)$ and $50^{\circ} \mathrm{C}(O)$. The lines are fits of Equation (8) to the data: $D_{\text {pull }} / D_{\text {pull, }, 0}=\exp \left(-R_{\mathrm{H}} / \xi\right)$.

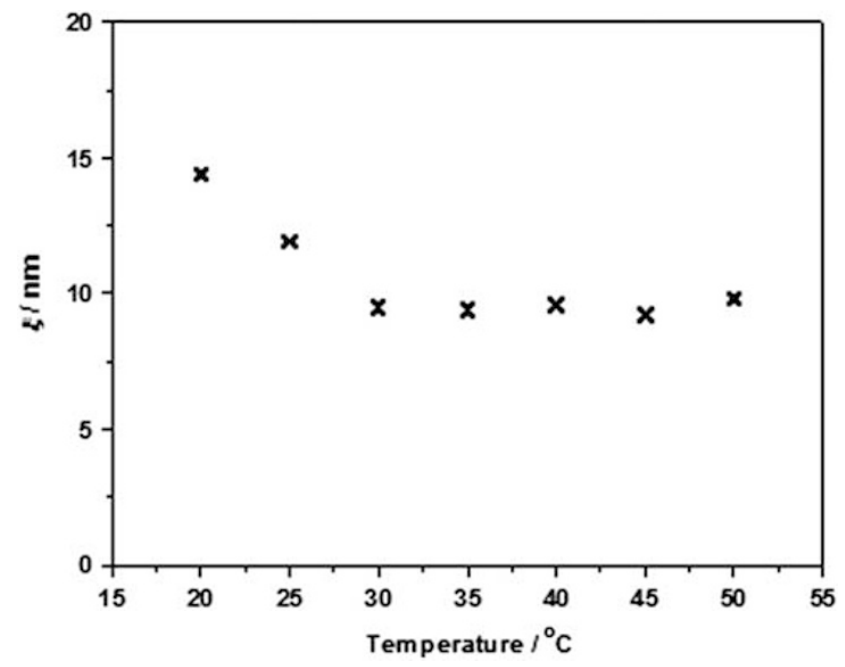

Figure 9 The temperature dependence of the hydrodynamic screening length, $\xi$, in a $1 \% \kappa$-carrageenan solution, estimated from a fit of Equation (8) to the results shown in Figure 8.

expressed as: ${ }^{25-27}$

$$
D_{\text {pull }} / D_{\text {pull }, 0}=\exp \left(-R_{\mathrm{H}} / \xi\right)
$$

where $R_{\mathrm{H}}$ is the hydrodynamic radius of the probe polymer and $\xi$ is the hydrodynamic screening length, considered to represent the hydrodynamic mesh size formed by the host polymer. Therefore, $\xi$ can be calculated by plotting the ratio $D_{\text {pull }} / D_{\text {pull, }, 0}$ as a function of $R_{\mathrm{H}}$.

Semi-logarithmic plots of the $D_{\text {pull }} / D_{\text {pull, } 0}$ vs $R_{\mathrm{H}}$ at various temperatures are shown in Figure 8. By Equation (8), the slope represents the inverse of $\xi$. Therefore, a decrease in the slope indicates an increase in $\xi$. The slopes were similar above $T_{\text {sg }}$ and became gentle with decreasing temperature below $T_{\mathrm{sg}}$. The estimated $\xi$ are summarized in Figure 9 and were almost constant with a value of $\sim 9.5 \mathrm{~nm}$ above $T_{\mathrm{sg}}$. $\xi$ increased with decreasing temperature below $T_{\mathrm{sg}}$ with 
values of $11.9 \mathrm{~nm}$ at $25^{\circ} \mathrm{C}$ and $14.4 \mathrm{~nm}$ at $20^{\circ} \mathrm{C}$. From this result, it is believed that the $\kappa$-carrageenan chains formed aggregates and enlarged the interspace among networks of the solute $\kappa$-carrageenan chains and the aggregates to increase $\xi$. This finding is in good agreement with the behavior of $I_{\text {kappa }}(0)$ shown in Figure 6 .

Note that the intercepts of the plot in Figure 8 were not equal to 1 and were shifted to higher values below $T_{\mathrm{sg}}$. This behavior is attributed to the differences in the local viscosity of the network interspace and the pure solvent. The local viscosity is increased by the solute $\kappa$-carrageenan chains that are smaller than the probe pullulan. These chains cannot contribute to the hydrodynamic restriction on the probe mobility as a network, but can increase the local viscosity. The increase of the intercept most likely indicates that the local viscosity has decreased by involving the smaller $\kappa$-carrageenan chains with the aggregation below $T_{\mathrm{sg}}$. However, the reason for a value greater than 1 is not well understood.

\section{CONCLUSIONS}

The mobility of pullulan with different molecular weights in a $1 \%$ $\kappa$-carrageenan solution has been studied using the pulsed field gradient stimulated spin-echo method. The diffusion behaviors of pullulan probes were elucidated in terms of the restriction effect of the hydrodynamic interaction. The diffusion coefficients of pullulan show a strong dependence on the geometry of the $\kappa$-carrageenan network. Furthermore, the hydrodynamic screening lengths, $\xi$, were calculated by fitting $D_{\text {pull }} / D_{\text {pull, }, 0}$ as a function of $R_{\mathrm{H}}$. Because the $\kappa$-carrageenan chains are involved in aggregation, $\xi$ increased markedly below $T_{\mathrm{sg}}$. It is expected that this methodology can be used to estimate $\xi$ in gels formed by polysaccharide aggregates.

\section{ACKNOWLEDGEMENTS}

We are thankful for the financial support of a Grant-in-Aid for Scientific Research (No. 21550203) from the Ministry of Education, Culture, Sports, Science and Technology, Japan (MECSST). The purchase of the NMR cooling system was supported by a Grant-in-Aid (No. 22240076) from MECSST.

1 Hoare, T. R. \& Kohane, D. S. Hydrogels in drug delivery: progress and challenges. Polymer 49, 1993 (2008).

2 Fieber, W., Herrmann, A., Ouali, L., Velazco, M. I., Kreutzer, G., Klok, H. A., Ternat, C., Plummer, C. J. G., Manson, J. A. E. \& Sommer, H. NMR diffusion and relaxation studies of the encapsulation of fragrances by amphiphilic multiarm star block copolymers. Macromolecules 40, 5372 (2007).
3 Gostan, T., Moreau, C., Juteau, A., Guichard, E. \& Delsuc, M. A. Measurement of aroma compound self-diffusion in food models by DOSY. Magn. Reson. Chem. 42, 496 (2004).

4 Matsukawa, S. in Food Hydrocolloids: Characteristics, Properties and Structures (ed. Hollingworth, C. S.) Ch. 5, 145 (Nova Science Pub Inc, New York, 1979).

5 Hikichi, K. Sol-gel transition of $\kappa$-carrageenan as viewed through NMR. Polym. Gels Networks 1, 19 (1993).

6 Glicksman, M. in Polysaccharides in Food: Gelling Hydrocolloids in Food Product Applications (eds Blanshard, J. M. V. \& Mitchell, J. R.) 185-204 (London, Butterworths, 1979).

7 Guo, J. H., Skinner, G. W., Harcum, W. W. \& Barnum, P. E. Investigating the fundamental effects of binders on pharmaceutical tablet performance. Drug. Dev. Ind. Pharm. 25, 1129 (1999).

8 De Ruiter, G. A. \& Rudolph, B. Carrageenan biotechnology. Trends Food Sci. Tech. 8 389 (1997).

9 Takemasa, M. \& Chiba, A. Gelation mechanism of $\kappa$ - and $\mathrm{u}$-carrageenan investigated by correlation between the strain-optical coefficient and the dynamic shear modulus. Macromolecules 34, 7427 (2001).

10 Mangione, M. R., Giacomazza, D., Bulone, D., Martorana, V. \& San Biagio, P. L. Thermoreversible gelation of $\kappa$-carrageenan: relation between conformational transition and aggregation. Biophys. Chem. 104, 95 (2003).

11 Zhang, Q. J. \& Matsukawa, S. Theoretical analysis of water ${ }^{1} \mathrm{H} T_{2}$ based on chemical exchange and polysaccharide mobility during gelation. Food Hydrocolloid 18, 441 (2004).

12 Dai, B. N. \& Matsukawa, S. NMR studies of the gelation mechanism and molecular dynamics in agar solutions. Food Hydrocolloid 26, 181 (2012).

13 McIntyre, D. D. \& Calgary, H. J. V. Structural studies of pullulan by nuclear magnetic resonance sepctroscopy. Starch 45, 406 (1993).

14 Tojo, E. \& Prado, J. A simple ${ }^{1} \mathrm{H}$ NMR method for the quantification of carrageenans in blends. Carbohydr. Polym. 53, 325 (2003).

15 Amsden, B. Solute diffusion in hydrogels. An examination of the retardation effect. Polym. Gels Networks 6, 13 (1998).

16 Mstsukawa, S., Sagae, D. \& Mogi, A. Molecular diffusion in polysaccharide gel systems as observed by NMR. Progr. Colloid. Polym. Sci. 136, 171 (2009).

17 Price, W. S. in NMR Studies of Translational Motion (Cambridge University Press, New York, 2009).

18 Einstein, A. in Investigation on the Theory of Brownian Motion 1-18 (Dover Publications Inc, New York, 1956).

19 Doi, M. \& Edward, S. F. in The Theory of Polymer Dynamics (Oxford University Press, New York, 1986).

20 Nishinari, K., Kohyama, K., Williams, P. A., Phillips, G. O., Burchard, W. \& Ogino, K. Solution properties of pullulan. Macromolecules 24, 5590 (1991)

21 Ying, Q. \& Chu, B. Overlap concentration of macromolecules in solution. Macromolecules 20, 362 (1987).

22 Kawahara, K., Ohta, K., Miyamoto, H. \& Nakamura, S. Preparation and solution properties of pullulan fractions as standard samples for water-soluble polymers. Carbohydr. Polym. 4, 335 (1984)

23 Takahashi, A., Sakai, M. \& Kato, T. Melting temperature of thermally reversible gel: $\varepsilon$ effect of branching on the sol-gel transition of polyethylene gels. Polym. J. 12, 335 (1980).

24 Shimizu, M., Brenner, T., Liao, R. Q. \& Matsukawa, S. Diffusion of probe polymer in gellan gum solutions during gelation process studied by gradient NMR. Food Hydrocolloid 26, 28 (2012).

25 Cukier, R. I. Diffusion of brownian spheres in semidilute polymer solutions. Macro molecules 17, 252 (1984).

26 De Gennes, P. G. Dynamics of entangled polymer solutions. II. Inclusion of hydrodynamic interactions. Macromolecules 9, 594 (1976).

27 Matsukawa, S. \& Ando, I. A study of self-diffusion of molecules in polymer gel by pulsed-gradient spin-echo ${ }^{1} \mathrm{H}$ NMR. Macromolecules 29, 7136 (1996). 